\title{
Bordelek hernia causing right ureteric obstruction with pyelonephritis
}

\author{
Masatomo Kanno ${ }^{1}$, Fumihiro Oshita ${ }^{2 *}$, Kuboi Yoriko ${ }^{1}$, Shinsuke Ueta ${ }^{1}$, Natsuki Kawata ${ }^{1}$, Yuichi Kurakami ${ }^{1}$, Mao Matsubayashi ${ }^{1}$, Keisuke \\ Iwabuchi $^{1}$, Yohsuke Kunishi ${ }^{1}$, Mitsuyasu Ohta ${ }^{1}$, Takeshi Watanabe ${ }^{3}$ and Kohichiro Yoshie ${ }^{1}$ \\ ${ }^{1}$ Department of General Medicine, Kanagawa Prefectural Ashigarakami-hospital, Matsudasouryo 866-1, Matsuda-machi, Kanagawa 258-0003, Japan \\ ${ }^{2}$ Department of Pulmonary Medicine, Kanagawa Prefectural Ashigarakami-hospital, Matsudasouryo 866-1, Matsuda-machi, Kanagawa 258-0003, Japan \\ ${ }^{3}$ Department of Urology, Kanagawa Prefectural Ashigarakami-hospital, Matsudasouryo 866-1, Matsuda-machi, Kanagawa 258-0003, Japan
}

\begin{abstract}
Bordelek hernia $(\mathrm{BH})$ is a congenital hernia of the diaphragm and is most asymptomatic. Besides, adult symptomatic $\mathrm{BH}$ on the right side is rare. We herein report a case of $\mathrm{BH}$ that caused pyelonephritis due to the right ureter obstruction. We detained a ureteric stent to the renal pelvis and treated pyelonephritis with ceftriaxone. The patient was relief from pyelonephritis in 3 days. We should note that pyelonephritis is caused by $\mathrm{BH}$ containing the ureter obstruction in the rare.
\end{abstract}

\section{Introduction}

Bordelek hernia $(\mathrm{BH})$ is a congenital hernia of the diaphragm with an overall prevalence in adults of between 0.17 to $10.5 \%$ [1-4], and is most asymptomatic. Besides, adult symptomatic $\mathrm{BH}$ on the right side is rare $[5,6]$. This is a first report that an elderly female complained pyelonephritis by obstruction of the right ureter due to $\mathrm{BH}$.

\section{Case report}

An 85 years old female administered to the emergency department with moderate back pain and chilling on a background of a month history of intermittent hematuria. She had hypertension and required medication. On examination, she had fever with a temperature of $37.5^{\circ} \mathrm{C}$. The blood pressure was $110 / 80 \mathrm{mmHg}$, the pulse 119 beats per minute, the respiratory rate 24 beats per minute. There was some mild flank tenderness on the right. Respiratory and abdominal examination was normal. There was no edema of the arms and legs. Laboratory data revealed that serum creatinine was elevated at $1.64 \mathrm{umol} / \mathrm{L}$, eGFR was $23.3 \mathrm{~mL} / \mathrm{L}$. Inflammatory markers were elevated such as white cell counts at $10,900 / \mu \mathrm{L}$ (reference range 4,000 to $8,000 / \mu \mathrm{L}$ ) and C-reactive protein at $5.1 \mathrm{mg} / \mathrm{dL}$ (reference range below $0.3 \mathrm{mg} / \mathrm{dL}$ ). Urinalysis revealed mild hematuria by dipstick, and bacteria with mild leukocytes were detected in urinary sediment. There were no abnormal signs of the abdomen on plain radiographs. A computed tomography (CT) scan demonstrated a $\mathrm{BH}$ of the right diaphragm. Right ureter partially was into the thorax through the hernia. The ureter was obstructed at the neck of hernia and was then dilated in the thorax. The right kidney located in abdomen, however the right pelvis was also dilated (Figure 1). There was no ureter dilatation below the hernia in the abdomen. We diagnosed that $\mathrm{BH}$ causing right ureteric obstruction with hydronephrosis and pyelonephritis. E. coli has been identified as causal bacteria in pyelonephritis. We detained a ureteric stent to the renal pelvis and treated pyelonephritis with ceftriaxone for 5 days, which was sensitive to $E$. coli. The patient was relief from symptoms in 3 days, and bacteria with leukocytes in urinary sediment disappeared at day 5 . A $\mathrm{CT}$ on a week later showed that the right pelvis and ureter returned to normal size (Figure 2). She was scheduled to have her stent changed every 6 to 12 months because of the risk of stent encrustation causing obstruction.

\section{Discussion}

Congenital diaphragmatic hernia occurs from incomplete closure between the pars lumbers and pars costalis parts of the diaphragm during fetal development [7]. The majority of them present during neonatal life with concomitant congenital other organs abnormalities. Symptomatic BH in adults is rare with approximately 100 cases reported. Asymptomatic BH occur equality on the right and left hand sides of the diaphragm $[3,4]$, however, symptomatic $\mathrm{BH}$ tend to occur on the left $[1,2]$. The contents of $\mathrm{BH}$ have included stomach, omentum, bowel, and others $[1,8]$. The presence of right renal pelvis or ureter within a $\mathrm{BH}$ is extremely rare with only four cases reported [6,9-11]; three presented flank or abdominal pain and another was symptomatic. The presence of pyelonephritis is only in our case. The $\mathrm{BH}$ in adults was presented from an incidental finding on imaging to strangulation of intra-abdominal contents with significant morbidity and mortality. Symptoms are often non-specific such as abdominal pain, dyspnea, chest pain and nausea. There was an asymptomatic case to be discovered incidentally. Diagnosis is ultimately via imaging and CT provides the most accurate and reliable method $[2,12]$. Discontinuity of the posterior diaphragm is usually clearly identified protrusion of intra-abdominal contents including ureter is clear. Importantly, CT scan identified associated complications of ureteric herniation including hydro-nephrosis and hydro-ureter, which is evidenced by

Correspondence to: Fumihiro Oshita, MD, Department of Pulmonary Medicine Kanagawa Prefectural Ashigarakami-hosptal, Matsudasouryo 866-1, Matsudamachi, Kanagawa 258-0003, Japan, Tel: +81-465-83-0351; Fax: +81-465-82 5377; E-mail: foshita@ashigarakami-hospital.jp

Key words: bordelek hernia, hydro-nephrosis, pyelonephritis, ureteric stent

Received: April 28, 2016; Accepted: May 13, 2016; Published: May 17, 2016 

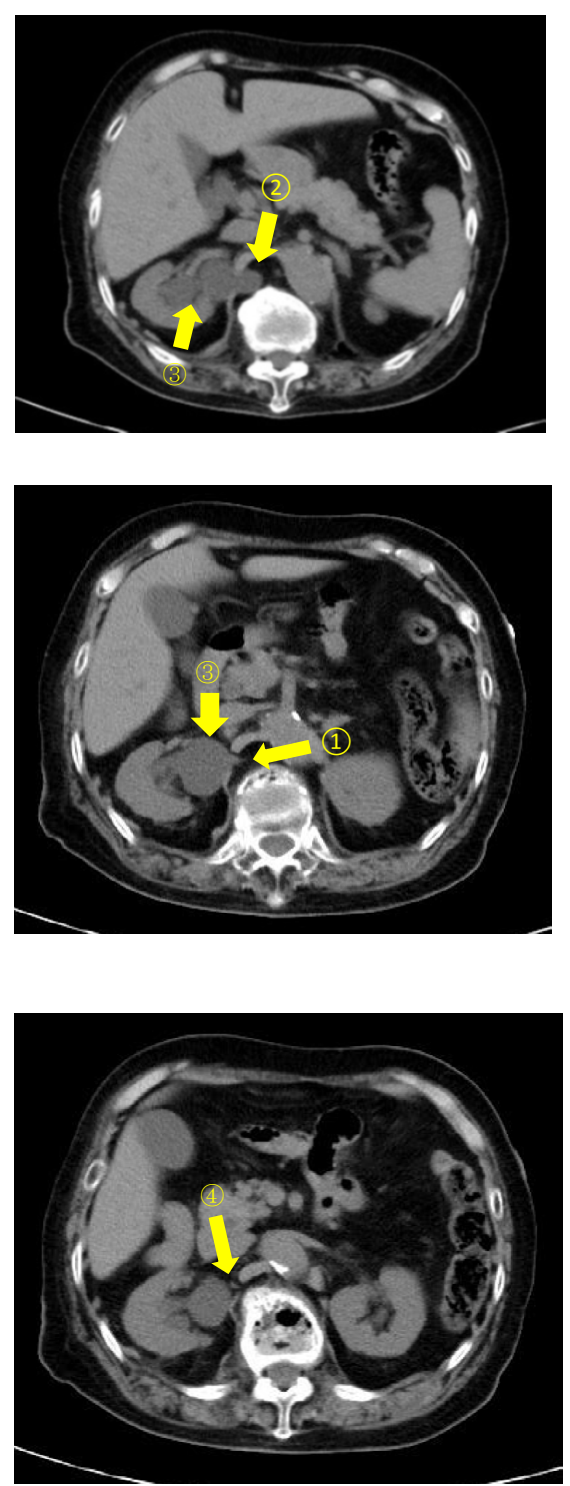

Figure 1. CT scan demonstrated that right ureter was obstructed at the neck of hernia (1)). The ureter above the neck of the hernia was distended in the thorax (2)). The right renal pelvis in abdominal cavity was also distended (3). There was no dilatation of the ureter below the hernia in the abdomen(4)).

dilation above the hernia neck and a normal calibrate ureter below. Of the reported cases on $\mathrm{BH}$ containing ureter, three were associated with dilated renal pelvis and ureter with similar CT findings to our case. Ultrasound, whilst able to identify hydro-nephrosis in our case, was not able to identify the hernia or the site of obstruction.

The optimal treatment of $\mathrm{BH}$ causing obstructive pyelonephritis is not established. We were able to success pass a stent through kinking of the ureter to the renal pelvis which provided immediate relief of the patient symptoms. Similarly, a patient with BH was able to be successfully passed a retrograde stent with resolution of hydronephrosis [10]. Unfortunately, our patient relapsed pyelonephritis after 3 months. Thus, definitive surgical correction of $\mathrm{BH}$ might be considered because of the risk of herniation and strangulation of abdominal viscera [8]. In particular, laparoscopic repair confers all the benefits of minimally invasive surgery. However, diaphragmatic surgery is not without complications, and our elderly patient was

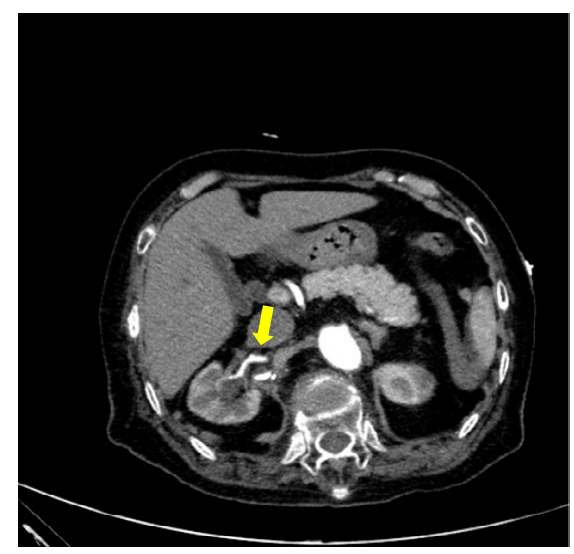

Figure 2. CT scan demonstrated right pelvis and ureter were returned to normal size by insertion of a stent.

not recommended because a minimal benefits as the risk of further herniation over their remaining lifetime. She is required regular CT imaging in order to check recurrence.

In conclusion, we described a case of an 85 year old female presenting with a right sided $\mathrm{BH}$ causing hydro-nephrosis and hydro-ureter with pyelonephritis. Symptomatic BH in adult patients is rare and there have been only four cases of $\mathrm{BH}$ containing ureter in the literature. Ureteric stent with antibiotics offers a safer alternative to surgical repair of the hernia with pyelonephritis and may be a more appropriate treatment in elderly patients. We should note that pyelonephritis is caused by $\mathrm{BH}$ containing the ureter obstruction in the rare.

\section{References}

1. Vithoulkas G (2012) The Science of Homeopathy. 6th ed. International Academy of Classical Homeopath., 2012.

2. Sloan A, Hussain I, Maqsood M, Eremin O, El-Sheemy M (2010) The effects of smoking on fracture healing. Surgeon 8: 111-116. [Crossref]

3. Zeeden H (1992) Symphytum. British Homeopathic Journal 81: 110.

4. Sakakura K, Neto R, Belluci M, Wenzel A, Scaf G, et al. (2008) Influence of homeopathic treatment with comfrey on bone density around titanium implants. A digital subtraction radiography study in rats. Clin Oral Implants Res 19. [Crossref]

5. Spin-Neto R, Belluci MM, Sakakura CE, Scaf G, Pepato MT, et al. (2010) Homeopathic Symphytum officinale increases removal torque and radiographic bone density around titanium implants in rats. Homeopathy 99: 249-254. [Crossref]

6. Oberbaum M, Yakovlev E, Kaufman D, Shoshan S (2004) Effect of Arnica montana and Symphytum officinalis on bone healing in guinea pigs. British Homeopathic Journal 83: 90

7. Vithoulkas G, Carlino S (2010) The "continuum" of a unified theory of diseases. Med Sci Monit 16: SR7-15. [Crossref]

8. Staiger C (2013) Comfrey root: from tradition to modern clinical trials. Wien Med Wochenschr 163: 58-64. [Crossref]

9. Boericke W (2008) Boericke's New Manual of Homeopathic Materia Medica with Repertory B. Jain Publishers (P) Ltd.

10. Allen TF (2011) The Encyclopedia of Pure Materia Medica. Narayana - Verlag.

Copyright: (C2016 Kanno M. This is an open-access article distributed under the terms of the Creative Commons Attribution License, which permits unrestricted use, distribution, and reproduction in any medium, provided the original author and source are credited. 